\title{
INFLUENCIA DEL DESEMPLEO EN MANDOS ARGENTINOS SOBRE SU POSICIONAMIENTO FRENTE A LA OBEDIENCIA
}

\section{The influence of unemployment on the norm of obedience}

\author{
Bernard Gangloff $^{1}$, Luisa Mayoral ${ }^{2}$ y María del Carmen Romero ${ }^{3}$
}

\section{RESUMEN}

La aprobación social de una conducta y el menosprecio de la conducta opuesta constituyen características típicas de las normas sociales. Se ha constatado, gracias a estudios recientemente realizados en la Provincia de Buenos Aires (Argentina), que los mandos con personal a cargo aprueban las conductas alineadas (es decir obedientes) entre sus subordinados -lo que permite pensar que se está en presencia de una norma social de alineación-, y que los subordinados son concientes de la existencia de esta norma.

Las preguntas que se plantean ahora son las siguientes 1) si los subordinados (aquí mandos medios sin personal a cargo) tienen conciencia de esta norma de manera global o si efectúan diferenciación entre sus diversas materializaciones, 2) si espontáneamente siguen estas normas en sus diversas materializaciones o si actúan de manera selectiva y 3) si estas actitudes y conductas globalizadoras o diferenciadoras varían en función de la ocupación efectiva o no de un puesto de trabajo.

Para responder a estos interrogantes, hemos utilizado el paradigma de la autopresentación sobrenormativa versus contranormativa. Esto es, hemos presentado a 180 mandos medios de la Provincia de Buenos Aires (la mitad de ellos con empleo y la otra mitad sin empleo) una lista de conductas para que ellos indiquen cuáles de ellas adoptarían acorde las diferentes consignas, a saber: espontánea, dar una buena imagen de sí mismos y dar una mala imagen de sí mismos.

En efecto, se observa que la percepción sobre el valor de la obediencia depende de la situación considerada, encontrándose una falta de clarividencia en cuanto a la valorización de las conductas obedientes por autocensura o por respeto a la legitimidad del superior jerárquico. Este aspecto surge con todas y cada una de las tres consignas utilizadas y también, aunque en menor medida, con independencia del hecho de estar actualmente con o sin empleo. Más allá de estos hallazgos concretos, se pone en evidencia la conveniencia de utilizar análisis del tipo ítem por ítem que, en oposición a los análisis globales, permiten evitar generalizaciones apresuradas.

Palabras claves: Obediencia; Empleo; Desempleo; Mandos medios.

\footnotetext{
${ }^{1}$ Laboratoire Parisien de Psychologie Sociale, Université Paris Ouest Nanterre La Défense, Francia. Correo electrónico: bernard.gangloff@ univ-rouen.fr

${ }^{2}$ Centro de Estudios en Administración. Universidad Nacional del Centro de la Provincia de Buenos Aires, Tandil, Argentina. Correo electrónico: mayoral.luisa@gmail.com

${ }^{3}$ Centro de Estudios en Administración. Universidad Nacional del Centro de la Provincia de Buenos Aires, Tandil, Argentina. Correo electrónico: romero@econ.unicen.edu.ar
} 


\begin{abstract}
The social value attributed to certain behaviour and the rejection towards its opposing behaviour characterise social norms. Recent studies carried out in Buenos Aires, Argentina, have shown that top-level chiefs approve of obedience o "aligned behaviour" among their subordinates. This evidences the existence of an obedience social norm that subordinates are aware of.

Thus three main questions arise: 1) Are mid-level chiefs (i.e. subordinates managers) conscious of this 'alignment' norm in a global or in a selective way?, 2) Is this norm followed spontaneously or deliberately? and 3) Does this behaviour vary depending on the subject being employed or unemployed?

The paradigm of spontaneous versus over-normative versus counter-normative self report was employed to find answers to these questions. One hundred and eighty mid-level chiefs were divided into three groups. Within these groups, half of them were employed, the other half were unemployed. Each group was asked to answer considering three different attitudes: the first group was asked to answer spontaneously, the second group was asked to answer considering a good impression wanted to be given, and the third group had to answer as if giving a bad impression of themselves was their intention. Each participant within these groups was presented with a list of options from which to choose how they would behave.

It was observed that aligned behaviour is valued depending on the given context. However, there is no consciousness of the norm when subordinates behave in that way due to selfcensorship or due to respect towards legitimacy. This specific aspect shows up in each and every paradigm presented: spontaneous, over-normative, counter-normative and also, even though to a lesser extent, independently of the fact of being employed or unemployed at the moment of the study. Further from these concrete findings, it becomes evident the need for an item-by-item analysis which avoids making quick generalizations.
\end{abstract}

Keywords: Obedience, employment, unemployment, mid-level chiefs

\title{
INTRODUCCIÓN
}

Toda situación de reclutamiento para un reclutador, puede traducirse en la cuestión siguiente: ¿el candidato posee un buen perfil? Sabemos después de mucho tiempo, que los aspectos esenciales de este perfil involucran más a las competencias sociales que a las competencias técnicas, esto es, el «saber hacer » del candidato. Este saber hacer está constituido por lo que llamamos la personalidad. Hoy por hoy existe un cuasi-consenso dentro de los psicólogos, en el sentido de considerar que esta personalidad puede describirse gracias a cinco grandes dimensiones, que son: la Energía, la Amabilidad, el Carácter concienzudo, la Estabilidad emocional y la Apertura de espíritu; es el modelo de los Cinco Grandes ( $c f$. McCrae y Costa, 1987, 1996 ; Goldberg, 1990, 1993 ; Rolland, 1994, p65; Caprara, Barbaranelli et Borgogni, 1997, p13).

Hemos observado entonces (Gangloff, 2000) que el candidato ideal es generalmente un candidato enérgico (es decir, dinámico y dominante), amable (capaz de prestar cooperación y ser cordial), conscienzudo (es decir, meticuloso y perseverante), emocionalmente estable (capaz de controlar sus emociones y sus impulsos), y de espíritu abierto (abierto a la cultura y a la experiencia). 
Por otro lado, recientemente ha surgido otro criterio respecto de este saber hacer, otro factor no ligado a la personalidad, sino de tipo más bien normativo, que ha restado importancia a cada uno de estos cinco factores, y aún a la consideración de la personalidad : la adhesión a la norma de alineación o de obediencia.

Esta adhesión se traduce en evitar todo tipo de conductas que cuestionen la jerarquía de poderes dentro de un sistema social constituido como tal (cf. para una revisión: Gangloff, 2002). El individuo que adhiera a esta norma será no solamente sumiso a esta jerarquía, sino que también sentirá una suerte de fidelidad, evitando toda conducta, en palabras o actos concretos, que pudieran hacerlo portador de cuestionamientos. En este sentido, los reclutadores prefieren sistemáticamente a un candidato no enérgico, no amable, no concienzudo, emocionalmente inestable o de espíritu cerrado, pero obediente, a otro que posea la personalidad ideal, pero que sea potencialmente rebelde (Gangloff y Huet, 2004). Finalmente, también se ha observado que los candidatos en un reclutamiento, o más globalmente los asalariados, son conscientes de esta preferencia, es decir, son conscientes de la valorización social de la obediencia ( $c f$. Gangloff, 2002).

Si bien la mayoría de los estudios anteriormente citados han sido realizados en Francia, investigaciones complementarias en Argentina arrojan resultados similares. Se ha establecido así que, en las empresas argentinas, los cuadros de mando con responsabilidad jerárquica prefieren trabajar con subordinados obedientes y no con subordinados potencialmente rebeldes. (Gangloff, Mayoral y Duringer, 2005). Asimismo, se ha constatado que los subordinados de los mandos jerárquicos poseen "clarividencia normativa" respecto de la obediencia (tomando la expresión de Py y Somat, 1991), es decir que son conscientes de la valorización social de la obediencia y que espontáneamente se muestran obedientes antes que rebeldes (Mayoral, Gangloff y Duringer, 2005).

Sin embargo, la obediencia puede concretarse en diferentes conductas y a raíz de diferentes causas. Así, hemos creído necesario afinar los resultados anteriormente obtenidos, estudiando por una parte la posibilidad de establecer diferenciaciones en este sentido, y por la otra examinar si estas eventuales diferenciaciones varían en función de la ocupación efectiva o no de un puesto de trabajo. Este es el objeto del presente trabajo, realizado sobre una población de mandos medios argentinos. Tres son los interrogantes centrales de esta investigación : explorar 1) si los mandos medios son conscientes de esta norma de manera global o general o si efectúan diferenciaciones entre sus diversas materializaciones, 2) si, espontáneamente, ellos siguen esta norma en sus diferentes concretizaciones o si actúan de manera selectiva, y 3) si estas actitudes globalizadoras o diferenciadoras varían en función de la ocupación efectiva o no de un puesto de trabajo.

\section{MÉTODO}

\subsection{Participantes}

Nuestro estudio ha sido efectuado en una población de 180 mandos medios de la Provincia de Buenos Aires, de sexo masculino, de los cuales la mitad poseía empleo y la otra mitad estaba desempleada (dos grupos de 90 personas cada uno).

\subsection{Instrumento}

Los sujetos de cada uno de esos dos grupos fueron invitados a responder a un cuestionario de alineación ya empleado en Francia (Gangloff y Caboux, 2003) constituido por 12 ítems (cf. 
anexo). Pero, en cada uno de esos dos grupos, se constituyeron tres sub-grupos de treinta sujetos: en el primero los sujetos debían responder a un cuestionario tratando de dar su mejor imagen, en el segundo debían responder de manera espontánea, y en el tercero los sujetos debían responder intentando mostrarse con su peor imagen. La distribución de los sujetos en cada uno de los grupos, figura en la Tabla 1 a continuación:

Tabla 1: Distribución de mandos medios en función de su situación (con empleo / sin empleo) y de la consigna

\begin{tabular}{|l|c|c|c|}
\hline & $\begin{array}{c}\text { Consigna } \\
\text { Sobrenormativa } \\
(\mathbf{N}=\mathbf{6 0})\end{array}$ & $\begin{array}{c}\text { Consigna } \\
\text { Honesta } \\
(\mathbf{N}=60)\end{array}$ & $\begin{array}{c}\text { Consigna } \\
\text { Contranormativa } \\
(\mathbf{N}=60)\end{array}$ \\
\hline $\begin{array}{l}\text { Mandos medios con empleo } \\
(\mathbf{N}=90)\end{array}$ & $\mathrm{N}=30$ & $\mathrm{~N}=30$ & $\mathrm{~N}=30$ \\
\hline $\begin{array}{l}\text { Mandos medios desempleados } \\
(\mathbf{N}=90)\end{array}$ & $\mathrm{N}=30$ & $\mathrm{~N}=30$ & $\mathrm{~N}=30$ \\
\hline
\end{tabular}

\subsection{Procedimiento}

En cuanto a las tres consignas, he aquí, más precisamente, los textos utilizados:

- Consigna sobrenormativa (CS):

"A continuación, le presentamos un cuestionario que incluye doce afirmaciones.

Le pedimos que lo complete como si se tratara de una planilla que se adjunta a su C.V., que va a ser presentada a un reclutador que decidirá si lo selecciona o no para ocupar un puesto de mando en la empresa. Le solicitamos que indique si está de acuerdo o no con lo que dice cada una de las afirmaciones marcando con una cruz en la casilla «De acuerdo» 0 «En desacuerdo».

Finalmente, le pedimos que al responder trate de dar su mejor imagen como si se tratara de caer bien al reclutador y ser así contratado".

- Consigna honesta $(\mathrm{CH})$ :

"A continuación, le presentamos un cuestionario que incluye doce afirmaciones.

Le pedimos que lo complete como si se tratara de una planilla que se adjunta con su C.V., que va a ser presentada a un reclutador que decidirá si lo selecciona o no para ocupar un puesto de mando en la empresa. Le solicitamos que indique si está de acuerdo o no con lo que dice cada una de las afirmaciones marcando con una cruz en la casilla «De acuerdo»0 «En desacuerdo». Finalmente, le pedimos que responda de la manera más honesta posible".

- Consigna contranormativa (CC):

"A continuación, le presentamos un cuestionario que incluye doce afirmaciones.

Le pedimos que lo complete como si se tratara de una planilla que se adjunta a su C.V., que va a ser presentada a un reclutador que decidirá si lo selecciona o no para ocupar un puesto de mando en la empresa. Le solicitamos que indique si está de acuerdo o no con lo que dice cada una de las afirmaciones marcando con una cruz en la casilla «De acuerdo» «En desacuerdo».

Finalmente, le pedimos que trate de dar su peor imagen como si se tratara de no caer bien al reclutador, de modo de no ser contratado". 


\subsection{Análisis}

Se forman 6 grupos constituídos por mandos medios que se difierencian de acuerdo a la consigna (sobrenormativa, honesta, contranormativa) y por el hecho de tener o no un empleo: Sobrenormativa Mandos Con empleo (SMC), Sobrenormativa Mandos Sin empleo (SMS), Honesta Mandos Con empleo (HMC), Honesta Mandos Sin empleo (HMS), Contranormativa Mandos Con empleo (CMC), Contranormativa Mandos Sin empleo (CMS).

Tres tipos de análisis se realizaron sobre estos grupos. En primer lugar, se estudia si, dentro de cada grupo, las respuestas difieren dependiendo del ítem que se examina. En segundo lugar se pretende averiguar, en cada uno de los grupos y para cada ítem, si las respuestas difieren de la media teórica. Finalmente, se observan las eventuales diferencias entre los grupos, ítem por ítem.

\section{RESULTADOS}

\subsection{Diferencias entre los ítems dentro del grupo}

Nuestro primer análisis ha consistido en calcular las tasas de acuerdo/desacuerdo obtenidas en cada uno de los ítems, para examinar luego las similitudes (versus las diferencias) entre estas proporciones, comparando los ítems de a pares. Estadísticamente hablando, hemos utilizado el test de diferencia de proporciones de McNemar para datos apareados ${ }^{4}$.

De la lectura de las Tablas 2 a 7, surge que de 66 diferencias posibles, se tienen 30 diferencias para el grupo SMC, 32 para el grupo SMS, 29 para el grupo HMC, 24 para el grupo HMS, 6 para el grupo CMC y ninguna para el grupo CMS.

Esto significa, que como habíamos establecido en nuestra hipótesis de trabajo, existe una diferenciación en las respuestas según los ítems, difererenciación que varía en función de la consigna y de la condición frente al empleo. Particularmente, se observa que las consignas sobrenormativa y honesta presentan más diferencias que las consignas contranormativas.

Teniendo en cuenta que cada ítem puede conducir a 11 diferencias significativas, encontramos que los ítems que difieren significativamente con más de la mitad son los siguientes: dentro del grupo SMC los ítems 10, 12, 3 y 8, dentro del grupo SMS los ítems 10, 3, 2, 12 y 7, dentro del grupo HMC los ítems 10, 7 y 2, dentro del grupo HMS los ítems 10 y 7 . Esto significa que el ítem que se distingue claramente del resto es el ítem 10 (que corresponde a la aceptación del control de los métodos de trabajo) y que éste conduce sistemáticamente a una respuesta de aceptación. Es más, el grupo CMS, rehusa rechazar este control.

Conviene, antes de ir más lejos en este análisis, preguntarse si la variación en el número de diferencias observadas significa o no una polarización de las respuestas en relación a la proporción media teórica. Esta cuestión es abordada en el punto 2.

\footnotetext{
4 En la aplicación de este test es necesario considerar, no sólo la proporción de individuos que formularon su acuerdo a ambos ítems, sino también la proporción de individuos que formularon su acuerdo a una de las cuestiones pero no a la otra. En consecuencia, dos cuestiones pueden llegar a tener la misma proporción de acuerdos sin que por eso, ambas se diferencien de una tercera cuestión (así, por ej., en la Tabla 4, los ítems 4 y 9 presentan proporciones idénticas, pero sólo el ítem 4 llega a una diferencia significativa en relación al 7).
} 
Tabla 2: Valores de $\mathrm{p}$ obtenidos en caso de diferencias significativas (i.e. <.05) entre las respuestas para los 12 ítems del cuestionario realizado a los mandos medios con empleo, bajo la consigna sobrenormativa (Sobrenormativa Mandos Con empleo). Se agregan las cantidades de acuerdos y porcentajes obtenidos para cada respuesta).

\begin{tabular}{|l|l|l|l|l|l|l|l|c|c|c|c|c|}
\hline \multicolumn{1}{|c|}{ SMC } & \multirow{2}{*}{ R1 } & R2 & R3 & R4 & R5 & R6 & R7 & R8 & R9 & R10 & R11 & R12 \\
\hline R1 $(23 ; 77 \%)$ & & $<.01$ & & & & & .013 & & .031 & & $<.01$ \\
\hline R2 $(27 ; 90 \%)$ & & & $<.01$ & & $<.01$ & & & $<.01$ & $<.01$ & & & $<.01$ \\
\hline R3 $(12 ; 40 \%)$ & & & & .013 & & $<.01$ & $<.01$ & & & $<.01$ & .013 & \\
\hline R4 $(22 ; 73 \%)$ & & & & & & & & & & .039 & & $<.01$ \\
\hline R5 $(15 ; 50 \%)$ & & & & & & & $<.01$ & & & $<.01$ & & \\
\hline R6 $(23 ; 77 \%)$ & & & & & & & & .013 & & & & $<.01$ \\
\hline R7 $(27 ; 90 \%)$ & & & & & & & & $<.01$ & $<.01$ & & & $<.01$ \\
\hline R8 $(13 ; 43 \%)$ & & & & & & & & & & $<.01$ & .022 & \\
\hline R9 $(17 ; 57 \%)$ & & & & & & & & & & $<.01$ & & .012 \\
\hline R10 $(29 ; 97 \%)$ & & & & & & & & & & & .039 & $<.01$ \\
\hline R11 $(22 ; 73 \%)$ & & & & & & & & & & & & $<.01$ \\
\hline R12 $(8 ; 27 \%)$ & & & & & & & & & & & & \\
\hline
\end{tabular}

Tabla 3: Valores de $\mathrm{p}$ obtenidos en caso de diferencias significativas (i.e. <.05) entre las respuestas para los 12 ítems del cuestionario realizado a los mandos medios sin empleo, bajo la consigna sobrenormativa (Sobrenormativa Mandos Sin empleo). Se agregan las cantidades de acuerdos y porcentajes obtenidos para cada respuesta).

\begin{tabular}{|c|c|c|c|c|c|c|c|c|c|c|c|c|}
\hline SMS & R1 & $\mathbf{R 2}$ & R3 & R4 & R5 & R6 & R7 & R8 & R9 & R10 & R11 & R12 \\
\hline R1 $(17 ; 57 \%)$ & & & $<.01$ & & .039 & & & & & $<.01$ & & $<.01$ \\
\hline R2 $(25 ; 83 \%)$ & & & $<.01$ & & $<.01$ & $<.01$ & & $<.01$ & $<.01$ & & .039 & $<.01$ \\
\hline R3 $(4 ; 13 \%)$ & & & & $<.01$ & & .021 & $<.01$ & & .021 & $<.01$ & $<.01$ & \\
\hline $\mathbf{R 4}(19 ; 63 \%)$ & & & & & .012 & & & & & $<.01$ & & $<.01$ \\
\hline $\mathbf{R 5}(10 ; 33 \%)$ & & & & & & & $<.01$ & & & $<.01$ & & \\
\hline R6 $(12 ; 40 \%)$ & & & & & & & $<.01$ & & & $<.01$ & & \\
\hline R7 $(24 ; 80 \%)$ & & & & & & & & $<.01$ & $<.01$ & & & $<.01$ \\
\hline $\mathbf{R 8}(11 ; 37 \%)$ & & & & & & & & & & $<.01$ & & \\
\hline R9 $(12 ; 40 \%)$ & & & & & & & & & & $<.01$ & & \\
\hline $\mathbf{R 1 0}(28 ; 93 \%)$ & & & & & & & & & & & .013 & $<.01$ \\
\hline R11 $(18 ; 60 \%)$ & & & & & & & & & & & & $<.01$ \\
\hline $\mathbf{R 1 2}(7 ; 23 \%)$ & & & & & & & & & & & & \\
\hline
\end{tabular}


Tabla 4: Valores de $\mathrm{p}$ obtenidos en caso de diferencias significativas (i.e. <.05) entre las respuestas para los 12 ítems del cuestionario realizado a los mandos medios con empleo, bajo la consigna honesta (Honesta Mandos Con empleo). Se agregan las cantidades de acuerdos y porcentajes obtenidos para cada respuesta.

\begin{tabular}{|l|l|l|l|l|l|l|l|l|l|l|l|l|}
\hline \multicolumn{1}{|c|}{ HMC } & R1 & R2 & R3 & R4 & R5 & R6 & R7 & R8 & R9 & R10 & R11 & R12 \\
\hline R1 $(12 ; 40 \%)$ & & $<.01$ & & & & & .013 & & & $<.01$ & & \\
\hline R2 $(24 ; 80 \%)$ & & & $<.01$ & $<.01$ & $<.01$ & $<.01$ & & $<.01$ & $<.01$ & & $<.01$ & $<.01$ \\
\hline R3 $(8 ; 27 \%)$ & & & & & & & $<.01$ & & & $<.01$ & & \\
\hline R4 $(11 ; 37 \%)$ & & & & & & & $<.01$ & & & $<.01$ & & \\
\hline R5 $(9 ; 30 \%)$ & & & & & & & $<.01$ & & & $<.01$ & & \\
\hline R6 $(7 ; 23 \%)$ & & & & & & & $<.01$ & & & $<.01$ & & \\
\hline R7 $(22 ; 73 \%)$ & & & & & & & & $<.01$ & .013 & & .021 & $<.01$ \\
\hline R8 $(6 ; 20 \%)$ & & & & & & & & & & $<.01$ & .039 & \\
\hline R9 $(11 ; 37 \%)$ & & & & & & & & & & $<.01$ & & \\
\hline R10 $(25 ; 83 \%)$ & & & & & & & & & & $<.01$ & $<.01$ \\
\hline R11 $(14 ; 47 \%)$ & & & & & & & & & & & & .039 \\
\hline R12 $(6 ; 20 \%)$ & & & & & & & & & & & \\
\hline
\end{tabular}

Tabla 5: Valores de $\mathrm{p}$ obtenidos en caso de diferencias significativas (i.e. <.05) entre las respuestas para los 12 ítems del cuestionario realizado a los mandos medios sin empleo, bajo la consigna honesta (Honesta Mandos Sin empleo). Se agregan las cantidades de acuerdos y porcentajes obtenidos para cada respuesta.

\begin{tabular}{|l|l|l|l|l|l|l|l|l|l|l|l|l|}
\hline \multicolumn{1}{|c|}{ HMS } & R1 & R2 & R3 & R4 & R5 & R6 & R7 & R8 & R9 & R10 & R11 & R12 \\
\hline R1 $(14 ; 47 \%)$ & & & & & & & $<.01$ & & & $<.01$ & & \\
\hline R2 $(18 ; 60 \%)$ & & & $<.01$ & & & & .012 & .031 & & $<.01$ & & .022 \\
\hline R3 $(7 ; 23 \%)$ & & & & .021 & & & $<.01$ & & & $<.01$ & & \\
\hline R4 $(15 ; 50 \%)$ & & & & & & & $<.01$ & & & $<.01$ & & \\
\hline R5 $(13 ; 43 \%)$ & & & & & & & $<.01$ & & & $<.01$ & & \\
\hline R6 $(12 ; 40 \%)$ & & & & & & & $<.01$ & & & $<.01$ & & \\
\hline R7 $(27 ; 90 \%)$ & & & & & & & & $<.01$ & $<.01$ & & $<.01$ & $<.01$ \\
\hline R8 $(8 ; 27 \%)$ & & & & & & & & & & $<.01$ & & \\
\hline R9 $(13 ; 43 \%)$ & & & & & & & & & & $<.01$ & & \\
\hline R10 $(28 ; 93 \%)$ & & & & & & & & & & & $<.01$ & $<.01$ \\
\hline R11 $(12 ; 40 \%)$ & & & & & & & & & & & & \\
\hline R12 $(9 ; 30 \%)$ & & & & & & & & & & & \\
\hline
\end{tabular}


Tabla 6: Valores de $\mathrm{p}$ obtenidos en caso de diferencias significativas (i.e. <.05) entre las respuestas para los 12 ítems del cuestionario realizado a los mandos medios con empleo, bajo la consigna contranormativa (Contranormativa Mandos Con empleo). Se agregan las cantidades de acuerdos y porcentajes obtenidos para cada respuesta.

\begin{tabular}{|c|c|c|c|c|c|c|c|c|c|c|c|c|}
\hline \multirow{2}{*}{\multicolumn{13}{|c|}{$\begin{array}{c}\text { CMC } \\
\text { R1 }(11: 37 \%)\end{array}$}} \\
\hline & & & & & & & & & & & & \\
\hline R2 $(5 ; 17 \%)$ & & & $<.01$ & & & .013 & & & & & & $<.01$ \\
\hline R3 $(16 ; 53 \%)$ & & & & & & & & & & & .013 & \\
\hline R4 $(13 ; 43 \%)$ & & & & & & & & & & & & \\
\hline R5 $(12 ; 40 \%)$ & & & & & & & & & & & & \\
\hline R6 $(15 ; 50 \%)$ & & & & & & & & & & & .013 & \\
\hline R7 $(11 ; 37 \%)$ & & & & & & & & & & & & \\
\hline R8 $(10 ; 33 \%)$ & & & & & & & & & & & & \\
\hline R9 $(11 ; 37 \%)$ & & & & & & & & & & & & \\
\hline $\mathbf{R 1 0}(8 ; 27 \%)$ & & & & & & & & & & & & \\
\hline R11 $(5 ; 17 \%)$ & & & & & & & & & & & & $<.01$ \\
\hline $\mathbf{R 1 2}(16 ; 53 \%)$ & & & & & & & & & & & & \\
\hline
\end{tabular}

Tabla 7: Valores de $\mathrm{p}$ obtenidos en caso de diferencias significativas (i.e. <.05) entre las respuestas para los 12 ítems del cuestionario realizado a los mandos medios sin empleo, bajo la consigna contranormativa (Contranormativa Mandos Sin empleo). Se agregan las cantidades de acuerdos y porcentajes obtenidos para cada respuesta.

\begin{tabular}{|c|c|c|c|c|c|c|c|c|c|c|c|c|}
\hline \multirow{2}{*}{\multicolumn{13}{|c|}{$\begin{array}{c}\text { CMS } \\
\text { R1 }(13 ; 43 \%)\end{array}$}} \\
\hline & & & & & & & & & & & & \\
\hline $\mathbf{R 2}(12 ; 40 \%)$ & & & & & & & & & & & & \\
\hline R3 $(14 ; 47 \%)$ & & & & & & & & & & & & \\
\hline R4 $(11 ; 37 \%)$ & & & & & & & & & & & & \\
\hline R5 $(12 ; 40 \%)$ & & & & & & & & & & & & \\
\hline R6 $(11 ; 37 \%)$ & & & & & & & & & & & & \\
\hline R7 $(15 ; 50 \%)$ & & & & & & & & & & & & \\
\hline $\mathbf{R 8}(8 ; 27 \%)$ & & & & & & & & & & & & \\
\hline R9 $(13 ; 43 \%)$ & & & & & & & & & & & & \\
\hline $\mathbf{R 1 0}(17 ; 57 \%)$ & & & & & & & & & & & & \\
\hline $\mathbf{R 1 1}(10 ; 33 \%)$ & & & & & & & & & & & & \\
\hline $\mathbf{R 1 2}(12 ; 40 \%)$ & & & & & & & & & & & & \\
\hline
\end{tabular}

\subsection{Diferencias respecto de la proporción media teórica}

Nos hemos preguntado ahora si las respuestas de los sujetos difieren de la proporción media teórica -dicha proporción tiene el valor 0.5 y significa que la mitad de las personas del grupo está de acuerdo y que la otra mitad no lo está - y hemos efectuado esta comparación en base al test exacto de Fisher. Los resultados detallados figuran en la Tabla 8 y se resumen en la Tabla 9. Algunos hallazgos son dignos de remarcar:

- en el caso de los mandos medios con empleo, se observan 2 ítems donde las respuestas son superiores a la proporción media teórica, tanto en la consigna neutra u honesta como en la consigna sobrenormativa: los ítems 2 y 10, que corresponden a la ejecución sistemática de órdenes y a la aceptación del control de los métodos de trabajo. Por otro lado, bajo consigna 
contranormativa, el ítem 2 produce respuestas inferiores a la proporción media teórica. Esto significa que en esta población, el ítem 2 es el que produce la más fuerte variación entre consignas, y que por tanto, constituye el ítem con mayor clarividencia normativa.

- en el caso de los mandos medios sin empleo, se obtienen respuestas superiores a la proporción media teórica para los ítems 7 (evitar crear problemas) y 10, al igual que en la consigna sobrenormativa y en la consigna honesta.

Tabla 8: Número de acuerdos obtenidos por ítem. Se incluye el porcentaje entre paréntesis, para cada uno de los 6 grupos de mandos medios. En negrita se remarca el p-valor en relación a la proporción media teórica en caso de diferencias significativas.

\begin{tabular}{|c|c|c|c|c|c|c|c|c|c|c|c|c|}
\hline & 1 & 2 & 3 & 4 & 5 & 6 & 7 & 8 & 9 & 10 & 11 & 12 \\
\hline \multirow[t]{2}{*}{ SMC } & $\begin{array}{c}23 \\
(77 \%)\end{array}$ & $\begin{array}{c}27 \\
(90 \%)\end{array}$ & $\begin{array}{c}12 \\
(40 \%)\end{array}$ & $\begin{array}{c}22 \\
(73 \%)\end{array}$ & $\begin{array}{c}15 \\
(50 \%)\end{array}$ & $\begin{array}{c}23 \\
(77 \%)\end{array}$ & $\begin{array}{c}27 \\
(90 \%)\end{array}$ & $\begin{array}{c}13 \\
(43 \%)\end{array}$ & $\begin{array}{c}17 \\
(57 \%)\end{array}$ & $\begin{array}{c}29 \\
(97 \%)\end{array}$ & $\begin{array}{c}22 \\
(73 \%)\end{array}$ & $\begin{array}{c}8 \\
(27 \%)\end{array}$ \\
\hline & .03 & $<01$ & & & & .03 & $<01$ & & & $<01$ & & \\
\hline \multirow[t]{2}{*}{ SMS } & $\begin{array}{c}17 \\
(57 \%)\end{array}$ & $\begin{array}{c}25 \\
(83 \%)\end{array}$ & $\begin{array}{c}4 \\
(13 \%)\end{array}$ & $\begin{array}{c}19 \\
(63 \%)\end{array}$ & $\begin{array}{c}10 \\
(33 \%)\end{array}$ & $\begin{array}{c}12 \\
(40 \%)\end{array}$ & $\begin{array}{c}24 \\
(80 \%)\end{array}$ & $\begin{array}{c}11 \\
(37 \%)\end{array}$ & $\begin{array}{c}12 \\
(40 \%)\end{array}$ & $\begin{array}{c}28 \\
(93 \%)\end{array}$ & $\begin{array}{c}18 \\
(60 \%)\end{array}$ & $\begin{array}{c}7 \\
(23 \%)\end{array}$ \\
\hline & & $<01$ & $<.01$ & & & & .01 & & & $<01$ & & .03 \\
\hline \multirow[t]{2}{*}{ HMC } & $\begin{array}{c}12 \\
(40 \%)\end{array}$ & $\begin{array}{c}24 \\
(80 \%)\end{array}$ & $\begin{array}{c}8 \\
(27 \%)\end{array}$ & $\begin{array}{c}11 \\
(37 \%)\end{array}$ & $\begin{array}{c}9 \\
(30 \%)\end{array}$ & $\begin{array}{c}7 \\
(23 \%)\end{array}$ & $\begin{array}{c}22 \\
(73 \%)\end{array}$ & $\begin{array}{c}6 \\
(20 \%)\end{array}$ & $\begin{array}{c}11 \\
(37 \%)\end{array}$ & $\begin{array}{c}25 \\
(83 \%)\end{array}$ & $\begin{array}{c}14 \\
(47 \%)\end{array}$ & $\begin{array}{c}6 \\
(20 \%)\end{array}$ \\
\hline & & .01 & & & & .03 & & .01 & & $<01$ & & .01 \\
\hline \multirow[t]{2}{*}{ HMS } & $\begin{array}{c}14 \\
(47 \%) \\
\end{array}$ & $\begin{array}{c}18 \\
(60 \%)\end{array}$ & $\begin{array}{c}7 \\
(23 \%)\end{array}$ & $\begin{array}{c}15 \\
(50 \%)\end{array}$ & $\begin{array}{c}13 \\
(43 \%)\end{array}$ & $\begin{array}{c}12 \\
(40 \%)\end{array}$ & $\begin{array}{c}27 \\
(90 \%)\end{array}$ & $\begin{array}{c}8 \\
(27 \%)\end{array}$ & $\begin{array}{c}13 \\
(43 \%) \\
\end{array}$ & $\begin{array}{c}28 \\
(93 \%)\end{array}$ & $\begin{array}{c}12 \\
(40 \%)\end{array}$ & $\begin{array}{c}9 \\
(30 \%)\end{array}$ \\
\hline & & & .03 & & & & $<01$ & & & $<.01$ & & \\
\hline \multirow[t]{2}{*}{ CMC } & $\begin{array}{c}11 \\
(37 \%)\end{array}$ & $\begin{array}{c}5 \\
(17 \%)\end{array}$ & $\begin{array}{c}16 \\
(53 \%)\end{array}$ & $\begin{array}{c}13 \\
(43 \%)\end{array}$ & $\begin{array}{c}12 \\
(40 \%)\end{array}$ & $\begin{array}{c}15 \\
(50 \%)\end{array}$ & $\begin{array}{c}11 \\
(37 \%)\end{array}$ & $\begin{array}{c}10 \\
(33 \%)\end{array}$ & $\begin{array}{c}11 \\
(37 \%)\end{array}$ & $\begin{array}{c}8 \\
(27 \%)\end{array}$ & $\begin{array}{c}5 \\
(17 \%)\end{array}$ & $\begin{array}{c}16 \\
(53 \%)\end{array}$ \\
\hline & & $<01$ & & & & & & & & & $<.01$ & \\
\hline CMS & $\begin{array}{c}13 \\
(43 \%)\end{array}$ & $\begin{array}{c}12 \\
(40 \%)\end{array}$ & $\begin{array}{c}14 \\
(47 \%)\end{array}$ & $\begin{array}{c}11 \\
(37 \%)\end{array}$ & $\begin{array}{c}12 \\
(40 \%)\end{array}$ & $\begin{array}{c}11 \\
(37 \%)\end{array}$ & $\begin{array}{c}15 \\
(50 \%)\end{array}$ & $\begin{array}{c}8 \\
(27 \%)\end{array}$ & $\begin{array}{c}13 \\
(43 \%)\end{array}$ & $\begin{array}{c}17 \\
(57 \%)\end{array}$ & $\begin{array}{c}10 \\
(33 \%)\end{array}$ & $\begin{array}{c}12 \\
(40 \%)\end{array}$ \\
\hline
\end{tabular}

Tabla 9: Número de ítems, para los cuales los mandos medios expresan un acuerdo significativamente superior o inferior a la proporción media teórica.

\begin{tabular}{|c|c|c|c|}
\hline & & Mand & edios \\
\hline & & Con empleo & Sin empleo \\
\hline Sobrenormativa & Superior a la proporción media teórica & $1,2,6,7,10$ & $2,7,10$ \\
\hline & Inferior a la proporción media teórica & & 3,12 \\
\hline Honesta & Superior a la proporción media teórica & 2,10 & 7,10 \\
\hline Contranormativa & $\begin{array}{l}\text { Inferior a la proporción media teórica } \\
\text { Superior a la proporción media teórica }\end{array}$ & $6,8,12$ & 3 \\
\hline & Inferior a la proporción media teórica & 2,11 & \\
\hline
\end{tabular}

\subsection{Diferencias entre grupos por ítem}

Nuestro próximo interrogante es acerca de las eventuales diferencias de respuestas entre grupos, examen realizado en base al test exacto de Fisher.

La Tabla 10 muestra que son los ítems 10 (aceptación del control de los métodos de trabajo), 6 (la funcionalidad de la obediencia en términos de crecimiento laboral) y 7 (evitación de crear problemas) los que conducen a las variaciones más frecuentes 
La variable consigna permite constatar, entre la consigna sobrenormativa y la contranormativa, 8 diferencias significativas (sobre 12 posibles) para los mandos con empleo (SMC/CMC) y 6 para los mandos sin (SMS/CMS). Los mandos sin empleo manifiestan un poco menos de clarividencia en las diferentes materializaciones de la norma de obediencia.

Si mantenemos el foco en la condición frente al empleo (con empleo o sin empleo) se observa que respecto de la consigna sobrenormativa (SMC/SMS) se producen diferencias en las respuestas para los ítems 3 (autocensura) y 6, con tasas de aprobación más importantes para los que tienen empleo, y que respecto de la consigna contranormativa (CMC/CMS) se observan diferencias para el ítem 10, en la cual los porcentajes de acuerdo son más elevados para los desocupados.

Tabla 10: Valores de $\mathrm{p}$ en caso de diferencia significativa (i.e. cuando son inferiores a .05) entre los grupos, por ítem (así, entre SMC y SMS, para el ítem 3, la diferencia es significativa con un $\mathrm{p}=.02$ ).

\begin{tabular}{|c|c|c|c|c|c|c|c|c|c|c|c|c|c|}
\hline \multirow{2}{*}{ SMC } & & 1 & 2 & $\begin{array}{c}3 \\
02\end{array}$ & 4 & & & 7 & 8 & 9 & 10 & 11 & 12 \\
\hline & $\begin{array}{l}\text { SMS } \\
\text { HMC }\end{array}$ & $<.01$ & & & $<. t$ & & & & & & & .04 & \\
\hline & $\begin{array}{l}\text { CMC } \\
\text { C }\end{array}$ & $<.01$ & $<.01$ & & .0 & & & $<.01$ & & & $<.01$ & $<.01$ & .04 \\
\hline HMC & $\begin{array}{l}\text { CMS } \\
\text { HMS }\end{array}$ & & $<.01$ & $<.01$ & .0 & & & .01 & & $<.01$ & .04 & & \\
\hline IC & $\begin{array}{l}\text { CMC } \\
\text { CMS } \\
\text { CMS }\end{array}$ & & $<.01$ & .04 & & & .0 & $\begin{array}{c}\kappa .01 \\
\kappa .01\end{array}$ & & & $\begin{array}{l}<.01 \\
<.01 \\
.02\end{array}$ & .01 & $<.01$ \\
\hline
\end{tabular}

\section{DISCUSIÓN}

Las diferencias observadas entre consignas sobrenormativas y consignas contranormativas (es decir el hecho de que los mandos produzcan respuestas diferentes según que se les pida mostrar una buena imagen o una mala imagen) indican que existe una nítida clarividencia normativa. Sin embargo, estas diferencias no se dan en la totalidad de los ítems; esto significa, conforme a nuestra hipótesis, que esta clarividencia es selectiva. Se observa claramente (cf. Tabla 10) que los ítems 5 (autocensura) y 8 (el reconocimiento de la legitimidad del jefe) no son objeto de ninguna clarividencia, cualquiera sea la condición frente al empleo.

Este hallazgo tiene varias implicaciones. A menudo en los estudios que utilizan este tipo de cuestionarios, las respuestas de los participantes se consideran a nivel global, sin tener en cuenta los matices de los distintos ítems. Tal reduccionismo metodológico puede tener repercusiones importantes. Así, se puede arribar a generalizaciones teóricas apresuradas que "olvidan" que la teoría propuesta no es válida sino bajo ciertas condiciones y que en particular, ciertos ítems, relevantes en otras condiciones, no validan la proposición teórica (véase, por ejemplo Blasco, 1993, o Gangloff, 2008). En el presente caso, se observa que los cuadros entrevistados son conscientes del valor de la obediencia, pero que esta conciencia no se aplica a situaciones de valorización de la obediencia por autocensura o a situaciones de valorización de obediencia por respeto a la legitimidad del superior jerárquico. 
En este sentido, se observa que espontáneamente los mandos que hemos interrogado no responden de la misma manera a cada uno de los ítems, con 29 diferencias inter-ítems en el grupo HMC y 24 en el grupo HMS ( $c f$. Tablas 4 et 5).

Una vez más, lo dicho respecto del reduccionismo metodológico y sus implicaciones teóricas, se aplica plenamente. En el presente caso, significa que aún las respuestas obtenidas sin ningún condicionamiento de imagen (es decir, bajo la consigna honesta) también están condicionadas por las circunstancias particulares propuestas en los diferentes ítems.

Hemos constatado asimismo que estos resultados no varían sino débilmente, según se interrogue a los mandos con empleo o sin empleo, cuestión que no confirma entonces, más que débilmente, el efecto de a variable empleo. Así, en relación a esta clarividencia ( $c f$. Tabla 10), hemos encontrado sólo tres diferencias (dos en la consigna sobrenormativa, entre SMC y SMS; y una en la consigna contranormativa, entre CMC et CMS), y las diferencias inter-ítems ( $c f$. Tablas 2 a 7) dan resultados bastante homogéneos según el hecho de poseer o no un empleo. Esto significa que, respecto de los mandos medios, la interiorización de la norma de alineación es tal que resulta poco sensible a las pérdidas de empleo posteriores. Es decir que se trata de una interiorización o normalización resistente (otra podría ser la situación en los obreros y estamos realizando un estudio en este sentido para abordar esta cuestión). No obstante, las comparaciones con respecto a la proporción media teórica indican que los mandos sin empleo son, en consigna sobrenormativa, los únicos que proveen respuestas inferiores a la proporción media teórica, y que en consigna contranormativa son igualmente los únicos que no producen una respuesta inferior a la proporción media teórica. Es decir que la interiorización normativa de la obediencia, aunque importante, tanto en los mandos con empleo como sin empleo, no puede entonces, en esta segunda población, ser considerada como "perfecta".

Tal resultado confirma ciertas observaciones, según las cuales, la inserción laboral/profesional tendría, al menos en nuestras sociedades industrializadas, un objetivo no solamente económico sino también político, de perennización de las estructuras de poder, vía la conformidad social (ver Beauvois, 1984, o Gangloff, 1999). Por otra parte, y desde un plano meta-analítico, esto nos recuerda que la psicología social de las organizaciones posee ramificaciones y áreas de intersección con otras disciplinas de las ciencias humanas, ramificaciones y intersecciones que invitan a una aproximación sistémica (ver al respecto Vannereau, 2011).

\section{CONCLUSIONES}

Distintos estudios llevados a cabo en el ámbito laboral han puesto de relieve, en primer lugar que los empleados obedientes son valorados positivamente, y en segundo lugar que estos empleados son conscientes de esta valoración (véanse en particular, Gangloff, 2002; Gangloff, Mayoral y Duringer, 2005; Mayoral, Gangloff y Duringer, 2005). El presente estudio tuvo como objetivo profundizar estos resultados indagando acerca de si la conciencia de aquella valoración era efectivamente global o fluctuaba en función de diversas materializaciones de la obediencia canalizadas a partir de distintos ítems: en función de la consigna (dar un buena o mala imagen de sí mismo, o contestar lo más honestamente posible), y en función de la ocupación efectiva o no de un puesto de trabajo.

Los resultados muestran claramente un efecto consigna. Así pues, se observa que la clarividencia en términos de la valorización de la obediencia no es simple sino compleja, ya que 
depende de la situación dada; con una particular ausencia en cuanto a la valorización de las conductas de obediencia por autocensura o por respeto a la legitimidad del superior jerárquico.

También se observa que este aspecto diferencial opera en cada una de las tres consignas, y que también, aunque en menor medida, aparece con independencia de la situación de los encuestados de tener o no un empleo. Finalmente y más allá de estas observaciones sobre la clarividencia de las conductas obedientes, este estudio proporciona evidencia a favor de los análisis del tipo ítem por ítem, por oposición a los análisis de tipo global, permitiendo evitar generalizaciones apresuradas...

\section{REFERENCIAS}

Beauvois, J-L. (1984). La psychologie quotidienne. Paris: PUF.

Blasco, R.D. (1993). Validité et utilité de l'évaluation de la personnalité en gestion du personnel. Revue Européenne de Psychologie Appliquée, 43(3), 231-239.

Caprara, G.V., Barbaranelli, C. y Borgogni, L. (1997). Alter ego; les 5 facteurs fondamentaux de la personnalité. Paris: E.A.P.

Gangloff, B. (1999). Le bilan de compétences : une utopie nécessaire à l'anesthésie sociale. Psychologie et Psychométrie, Revue de Langue Française, 20(4), 67-85.

Gangloff, B. (2000). La saturation normative des tests dits de personnalité. $27^{\text {ème }}$ Congrès International de Psychologie, Stockholm (Suède).

Gangloff, B. (2002). L'internalité et l'allégeance considérées comme des normes: une revue. Les Cahiers de Psychologie Politique, $\mathrm{n}^{\circ}$ 2. [http://www.cahierspsypol.fr].

Gangloff, B. (2008). Normativité de la croyance en un monde juste via « le paradigme du législateur »: une étude sur des recruteurs. $14^{\text {ème }}$ Congrès International de Psychologie du Travail de Langue Française. (Hammamet, Tunisie, 2006). Lille : Ed. de l'AIPTLF, vol.4, 103-112.

Gangloff, B. y Caboux, N. (2003). Conformismo a la norma de alineación y reacciones a las injusticias profesionales. $29^{n o}$ Congreso Interamericano de Psicología. Lima, Perú.

Gangloff, B. y Huet, M. (2004). Poids respectif du gros cinq et de l'allégeant en situation de recrutement. $13^{\text {ème }}$ Congrès International de Psychologie du Travail et de Organisations, Bologne (Italie).

Gangloff, B., Mayoral, L. y Duringer, L. (2005). Los asalariados que prefieren los jefes argentinos: influencia del nivel de alineacion y del género, $30^{v o}$ Congreso Interamericano de Psicología, Buenos Aires (Argentine).

Goldberg, L.R. (1990). An alternative description of personality : the big-five factor. Journal of Personality and Social Psychology, 59, 1216-1229.

Goldberg, L.R. (1996). The structure of phenotypic personality traits. American Psychologist, 48(1), 26-34.

McCrae, R.R. y Costa, P.T. (1987). Validation of the five-factor model across instruments and observers. Journal of Personality and Social Psychology, 52, 81-90.

McCrae, R.R. y Costa, P.T. (1996). Toward a new generation of personality theories : theorical contexts for the five-factor model. In : J.S. Wiggins (Ed.), The five-factor model of personality : theorical perspectives. New-York: Guilford.

Mayoral, L., Gangloff, B. y Duringer, L. (2005). La percepcion de los mandos medios argentinos en cuanto al nivel de alineacion conveniente para ser reclutados, $30^{v o}$ Congreso Interamericano de Psicología, Buenos Aires (Argentine). 
Py, J. y Somat, A. (1991). Normativité, conformité et clairvoyance: leurs effets sur le jugement évaluatif dans un contexte scolaire. In: J-L. Beauvois, R-V. Joule y J-M. Monteil (Eds), Perpectives cognitives et conduites sociales. Cousset: Del Val, vol. 3, 167-193.

Rolland, J-P. (1994). Désirabilité sociale de marqueurs des dimensions de ma personnalité du modèle en 5 facteurs. Revue Européenne de Psychologie Apliquée, 44(1), 65-71.

Vannereau, J. (2011). Stratégies de modélisation des situations managériales par des élèves ingénieurs. In : L. Blot y M. L. Vitali (Eds), Modélisation et activités des ingénieurs. Paris: L'Harmattan, 257-288.

\section{Anexo: el cuestionario utilizado}

Este cuestionario comprende 9 ítems sobre las manifestaciones de la obediencia (items 1, 2, 3, 4, 5, 7, 9, 10 y 12) y 3 items sobre las razones de la obediencia (ítems 6, 8, 11). Se indica entre paréntesis y en negrita la dimensión presente en cada uno de los ítems (Esto no figuraba en el cuestionario presentado a los sujetos).

1) En el trabajo, me parece normal pedir sistemáticamente el acuerdo de mi jefe antes de tomar una decisión (abandono de toda autonomía decisoria).

2) En mi trabajo, me parece normal ejecutar todas las órdenes que me da mi superior (ejecución sistemática de órdenes).

3) Con mi jefe, intento raramente defender mis ideas (no defensa del propio punto de vista).

4) Cuando la dirección me comunica sus decisiones, es raro que yo discuta abiertamente (no cuestionamiento a las decisiones).

5) Evito hacer observaciones a mi superior, incluso si, en algunas ocasiones, se lo merece (autocensura).

6) Encuentro normal aceptar todas las decisiones de mis superiores si yo quiero subir en la escala jerárquica. (funcionalidad de la aceptación de decisiones de la autoridad en términos de crecimiento laboral).

7) Cualesquiera sean las condiciones de trabajo, encuentro normal evitar crear problemas (evitación de crear problemas).

8) Acepto más fácilmente un tono autoritario cuando este viene de mi jefe (reconocimiento de la legitimitad del jefe).

9) En todas las circunstancias, encuentro normal que la opinión de mi superior sea más importante que la mía (aceptación del predominio del punto de vista del jefe)

10) Encuentro normal que mi superior quiera verificar, no importa en qué momento, si yo respeto los métodos de trabajo (aceptación del control de los metodos de trabajo)

11) Me digo con frecuencia que mi patrón es el mejor ubicado para saber qué es lo bueno para su empresa (reconocimiento de la experiencia del jefe)

12) Encuentro normal que mi patrón no justifique sus decisiones ante mí, incluso si ellas me conciernen en forma directa (renuncia a pedir justificación por las decisiones). 
(c) (1)

Este texto está protegido por una licencia CreativeCommons $\underline{4.0}$.

Usted es libre para Compartir - copiar y redistribuir el material en cualquier medio o formato- y Adaptar el documento - remezclar, transformar y crear a partir del material- para cualquier propósito, incluso comercialmente, siempre que cumpla la condición de:

Atribución: Usted debe reconocer el crédito de una obra de manera adecuada, proporcionar un enlace a la licencia, e in- dicar si se han realizado cambios. Puede hacerlo en cualquier forma razonable, pero no de forma tal que sugiera que tie- ne el apoyo del licenciante o lo recibe por el uso que hace.

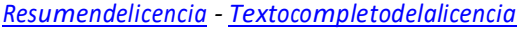

